\title{
A New Generalization of the Haddock Conjecture
}

\section{Songlin Xiao}

Academy of Intelligent Software, Guangzhou University, Guangdong Provincial Engineering Technology Research Center for Mathematical Educational Software

Guangzhou 510006, China

E-mail(corresp.): xiaosonglin2017@163.com

Received March 8, 2017; revised June 9, 2017; published online September 15, 2017

\begin{abstract}
In this paper, we establish some new criteria on the boundedness and asymptotic constancy of solutions for a class of scalar neutral functional differential equations with time-varying delays via differential inequality techniques. Our results are an improvement of existing ones and generalization of the Haddock conjecture.
\end{abstract}

Keywords: Haddock conjecture, neutral functional differential equation, time-varying delay, asymptotic constancy.

AMS Subject Classification: $34 \mathrm{C} 12 ; 39 \mathrm{~A} 11$.

\section{Introduction}

Consider the following scalar neutral functional differential equation (NFDE)

$$
[x(t)-p x(t-r)]^{\prime}=-F(x(t))+F(x(t-r)),
$$

where $F: \mathbb{R} \rightarrow \mathbb{R}$ is a continuous and strictly increasing function. In 1989 , Wu in [7] used the equation (1.1) to confirm the Haddock conjecture:

Conjecture [2]. Every solution of the delay differential equation

$$
[x(t)-p x(t-r)]^{\prime}=-x^{\frac{1}{3}}(t)+x^{\frac{1}{3}}(t-r),
$$

where $0<p<1, r>0$, tends to a constant as $t \rightarrow+\infty$.

Later on, variants of the above equations are used to model some population growth and spread of epidemics, and hence they have received considerable attention (see, for example, $[1,9,10,11,12,14]$ and the references therein). In particular, the asymptotic behavior of the following non-autonomous NFDE

$$
[x(t)-p x(t-r)]^{\prime}=H(t, x(t), x(t-r))
$$


has been deeply studied in $[3,8,13]$. Here, $H \in C\left(\mathbb{R}^{3}, \mathbb{R}\right)$ is periodic in its first argument, and locally Lipschitz continuous in its second argument. Note that $x^{\frac{1}{3}}$ is not locally Lipschitz continuous on $\mathbb{R}$, and the Haddock conjecture equations (1.1) and (1.2) can not be contained in (1.3). On the other hand, the delays in differential equations of population and ecology problems are usually time-varying in the real world, then equation (1.1) can be naturally generalized to the following non-autonomous NFDE with a time-varying delay:

$$
[x(t)-p x(t-\sigma(t))]^{\prime}=b(t)[-F(x(t))+F(x(t-\sigma(t)))],
$$

where $F \in C(\mathbb{R}, \mathbb{R})$ is strictly increasing, $\sigma, b \in C(\mathbb{R}, \mathbb{R})$ are bounded, and

$$
0<\sigma^{-}=\inf _{t \in \mathbb{R}} \sigma(t) \leq \sup _{t \in \mathbb{R}} \sigma(t)=\sigma^{+}, 0<b^{-}=\inf _{t \in \mathbb{R}} b(t) \leq \sup _{t \in \mathbb{R}} b(t)=b^{+} .
$$

Obviously, (1.1) and (1.2) are particular cases of (1.4) with $b(t)=1$ and $\sigma^{-}=$ $\sigma^{+}$.

It is well-known that a non-autonomous NFDE with a time-varying delay generally does not generate a semiflow and hence the methods for NFDE with constant delays in $[1,9,10,11,12,14]$ are not suitable for (1.4). Moreover, it should be mentioned that Propositions 4 and 5 of [6] played a crucial role in the discussions of its main results, but they are not true as demonstrated by a counterexample in [15]. Most recently, Liu [4,5] corrected these two propositions, and successfully used them to analyze the asymptotic behavior of some functional differential equations (see Propositions $4^{*}$ and $5^{*}$ in [5]). Therefore, the proof in [1] needs further improvement.

Thus a natural question arises: whether we can use the improved Proposition $4^{*}$ and Proposition $5^{*}$ of [5] to correct the proof of the main result of [7]. This is the main purpose of this paper.

Throughout this paper, we assume that

$$
F(0)=0, F(x) \text { is continuous differentiable on } \mathbb{R} \backslash\{0\}, F^{\prime}(x)>0,
$$

for all $x \in \mathbb{R} \backslash\{0\}$.

For convenience, we let $C=C\left(\left[-\sigma^{+}, 0\right], \mathbb{R}\right)$. If $\tau \geq 0, t_{0} \in \mathbb{R}$, and $x \in$ $C\left(\left[t_{0}-\sigma^{+}, t_{0}+\tau\right], \mathbb{R}\right)$, then, for any $t \in\left[t_{0}, t_{0}+\tau\right], x_{t} \in C$ is defined by $x_{t}(\theta)=x(t+\theta),-\sigma^{+} \leq \theta \leq 0$. Moreover, for $\varphi \in C$, we use $x_{t}\left(t_{0}, \varphi\right)$ $\left(x\left(t ; t_{0}, \varphi\right)\right)$ to denote the solution of (1.4) with the initial data $x_{t_{0}}\left(t_{0}, \varphi\right)=\varphi$.

The remaining part of this paper is organized as follows. In Section 2, we show the global existence of every solution for (1.4) with the initial data $x_{t_{0}}=\varphi \in C$. Based on the preparation in Section 2, we state and prove our main result in Section 3.

\section{Preliminaries}

In the following, assume that $G: \mathbb{R} \rightarrow \mathbb{R}$ is continuous and strictly increasing, and

$$
G(0)=0, G(x) \text { is continuous differentiable on } \mathbb{R} \backslash\{0\}, G^{\prime}(x)>0
$$


for all $x \in \mathbb{R} \backslash\{0\}$. Then, from Lemma 1, Proposition $4^{*}$ and Proposition $5^{*}$ in [5], we have

Proposition 1. Consider the differential equation,

$$
u^{\prime}=-G(u)+G(c+\varepsilon),
$$

where $c \neq 0$ is a given constant, $\varepsilon$ is a parameter satisfying $0 \leq \varepsilon \leq \frac{|c|}{2}$. Moreover, assume the initial condition

$$
u\left(t_{0}\right)=u_{0}, \quad\left(u_{0}<c\right) .
$$

Let $u=u\left(t ; t_{0}, u_{0}\right)$ be the solution of the initial value problems (2.1) and (2.2), and $\bar{\alpha}>0$ be a given constant. Then there exists a positive real number $\mu$ independent of $t_{0}$ and $\varepsilon$ such that

$$
(c+\varepsilon)-u\left(t ; t_{0}, u_{0}\right) \geq \mu>0 \quad \text { for } \quad t \in\left[t_{0}, t_{0}+\bar{\alpha}\right] .
$$

Proposition 2. Consider the differential equation,

$$
u^{\prime}=-G(u)+G(c-\varepsilon),
$$

where $c \neq 0$ is a given constant, $\varepsilon$ is a parameter satisfying $0 \leq \varepsilon \leq \frac{|c|}{2}$. Moreover, assume the initial condition

$$
u\left(t_{0}\right)=u_{0}, \quad\left(u_{0}>c\right) .
$$

Let $u=u\left(t ; t_{0}, u_{0}\right)$ be the solution of the initial value problems (2.3) and (2.4), and $\bar{\alpha}>0$ be a given constant. Then there exists a positive real number $\nu$ independent of $t_{0}$ and $\varepsilon$ such that

$$
u\left(t ; t_{0}, u_{0}\right)-(c-\varepsilon) \geq \nu>0 \quad \text { for } t \in\left[t_{0}, t_{0}+\bar{\alpha}\right] .
$$

Lemma 1. (see [4]). Let $t_{0} \in \mathbb{R}, T>0, \tilde{h} \in C\left(\left[t_{0}, t_{0}+T\right] \times \mathbb{R}, \mathbb{R}\right)$, and $\tilde{h}$ be nonincreasing with respect to the second variable. Then the initial value problem

$$
\frac{d x}{d t}=\tilde{h}(t, x), \quad x\left(t_{0}\right)=x_{0}
$$

admits a unique solution $x=x(t)$ on $\left[t_{0}, t_{0}+T\right]$.

Lemma 2. Let $\varphi \in C$. Then the solution $x_{t}\left(t_{0}, \varphi\right)$ of equation (1.4) exists and is unique on $\left[t_{0},+\infty\right)$.

Proof. Let $a(t)=p \varphi\left(t-\sigma(t)-t_{0}\right)$ and $D(t)=F\left(\varphi\left(t-\sigma(t)-t_{0}\right)\right)$ for $t \in$ $\left[t_{0}, t_{0}+\sigma^{-}\right]$. Consider the solution $y(t)$ of the following initial value problem,

$$
\left\{\begin{array}{l}
y^{\prime}(t)=b(t)[-F(y(t)+a(t))+D(t)], \\
y\left(t_{0}\right)=\varphi(0)-p \varphi\left(-\sigma\left(t_{0}\right)\right) .
\end{array}\right.
$$

By Lemma 1, $y(t)$ exists and is unique on $\left[t_{0}, t_{0}+\sigma^{-}\right]$. Denote $x(t)=y(t)+a(t)$. It follows that $x(t)-p \varphi\left(t-\sigma(t)-t_{0}\right)$ satisfies $(2.5)$, and $x_{t}(\varphi)$ exists and is unique on $\left[t_{0}, t_{0}+\sigma^{-}\right]$. Then $x_{t}(\varphi)$ exists and is unique on $\left[t_{0},+\infty\right)$ by induction.

By the proof of Lemma 3 in [7], we can take the following lemma: 
Lemma 3. Assume that $x \in C\left(\left(-\sigma^{+},+\infty\right), \mathbb{R}\right)$ is a bounded, and there exist constants $\bar{\delta}, \bar{p} \in \mathbb{R}$ such that $|\bar{p}|<1$ and $\lim _{t \rightarrow+\infty}[x(t)-\bar{p} x(t-\sigma(t))]=\bar{\delta}$, then $\lim _{t \rightarrow+\infty} x(t)=\bar{\delta} /(1-\bar{p})$.

\section{$3 \quad$ Boundedness and asymptotic constancy}

Theorem 1. Let $\varphi \in C$. Then $x\left(t ; t_{0}, \varphi\right)$ is bounded and tends to a constant as $t \rightarrow+\infty$.

Proof. Letting $x\left(t ; t_{0}, \varphi\right)=x\left(t_{0}-\sigma^{+} ; t_{0}, \varphi\right)=\varphi\left(-\sigma^{+}\right)$for $t \in\left[t_{0}-\sigma^{+}-\right.$ $\left.\sigma^{+}, t_{0}-\sigma^{+}\right]$, we can trivially extend $x\left(t ; t_{0}, \varphi\right)$ to $\left[t_{0}-\sigma^{+}-\sigma^{+},+\infty\right)$. For $t \geq t_{0}$, we denote

$$
\begin{aligned}
& x(t)=x\left(t ; t_{0}, \varphi\right), z(t)=\max _{t-\sigma^{+} \leq s \leq t}\{x(s)-p x(s-\sigma(s)),(1-p) x(s)\} \\
& v(t)=\min _{t-\sigma^{+} \leq s \leq t}\{x(s)-p x(s-\sigma(s)),(1-p) x(s)\} \\
& Q=\left\{t \mid t \in\left(t_{0}, \infty\right), z(t)=\max \{x(t)-p x(t-\sigma(t)),(1-p) x(t)\}\right\} .
\end{aligned}
$$

Now, we distinguish two cases to prove that $D^{+} z(t) \leq 0$ for all $t \geq t_{0}$.

Case 1. When $t \in\left(t_{0}, \infty\right) \backslash Q$. Then there exists $t^{*} \in\left[t-\sigma^{+}, t\right)$ such that

$$
\begin{array}{r}
z(t)=\max \left\{x\left(t^{*}\right)-p x\left(t^{*}-\sigma\left(t^{*}\right)\right),(1-p) x\left(t^{*}\right)\right\} \\
>\max \{x(t)-p x(t-\sigma(t)),(1-p) x(t)\} .
\end{array}
$$

From the continuity of $x(\cdot)$, we can choose a positive constant $\delta<\sigma^{+}$such that

$$
\max \{x(s)-p x(s-\sigma(s)),(1-p) x(s)\}<z(t) \text { for all } s \in[t, t+\delta],
$$

which yields

$$
\max \{x(s)-p x(s-\sigma(s)),(1-p) x(s)\} \leq z(t) \text { for all } s \in\left[t-\sigma^{+}, t+\delta\right] .
$$

We obtain

$$
\begin{aligned}
& z(t+h) \leq z(t) \text { for all } h \in(0, \delta) \\
& D^{+} z(t)=\limsup _{h \rightarrow 0^{+}} \frac{z(t+h)-z(t)}{h} \leq \limsup _{h \rightarrow 0^{+}} \frac{z(t)-z(t)}{h}=0 .
\end{aligned}
$$

Case 2. When $t \in Q$. We first claim that

$$
z(t)=(1-p) x(t)>x(t)-p x(t-\sigma(t)) \text { can not occur. }
$$

Otherwise,

$$
x(t-\sigma(t))>x(t) \text { and }(1-p) x(t-\sigma(t))>(1-p) x(t),
$$


which contradicts that

$$
z(t)=(1-p) x(t)=\max _{t-\sigma^{+} \leq s \leq t}\{x(s)-p x(s-\sigma(s)),(1-p) x(s)\} .
$$

So (3.1) is true, and thus we only consider the case that

$$
z(t)=x(t)-p x(t-\sigma(t)) \geq(1-p) x(t) .
$$

Then (1.4) implies that

$$
0 \leq[x(t)-p x(t-\sigma(t))]^{\prime} \leq b(t)[-F(x(t))+F(x(t))]=0 .
$$

Secondly, assume that

$$
z(s)=x(s)-p x(s-\sigma(s)) \text { for all } s \in\left(t, t+\frac{1}{2} \sigma^{-}\right] .
$$

Clearly, (3.2) gives us

$$
D^{+} z(t)=\limsup _{h \rightarrow 0^{+}} \frac{[x(t+h)-p x(t+h-\sigma(t+h))]-[x(t)-p x(t-\sigma(t))]}{h}=0 .
$$

Furthermore, suppose that there exists $s_{1} \in\left(t, t+\frac{1}{2} \sigma^{-}\right]$such that $z\left(s_{1}\right)>$ $x\left(s_{1}\right)-p x\left(s_{1}-\sigma\left(s_{1}\right)\right)$. From $(3.1)$, we can choose a constant $\tilde{T} \in\left[s_{1}-\sigma^{+}, s_{1}\right)$ such that

$$
z\left(s_{1}\right)=x(\tilde{T})-p x(\tilde{T}-\sigma(\tilde{T})) \geq(1-p) x(\tilde{T}) .
$$

This, together with the fact that $t-\sigma^{+}<s_{1}-\sigma^{+} \leq t+\frac{1}{2} \sigma^{-}-\sigma^{+}<t<s_{1}$, implies that

$$
x(\tilde{T})-p x(\tilde{T}-\sigma(\tilde{T})) \geq x(t)-p x(t-\sigma(t))=z(t) .
$$

We claim that

$$
x(\tilde{T})-p x(\tilde{T}-\sigma(\tilde{T}))=x(t)-p x(t-\sigma(t))=z(t) .
$$

Otherwise,

$$
x(\tilde{T})-p x(\tilde{T}-\sigma(\tilde{T}))>x(t)-p x(t-\sigma(t))=z(t) .
$$

Then $t<\tilde{T}<s_{1}$ and

$$
0=\left.[x(s)-p x(s-\sigma(s))]^{\prime}\right|_{s=\tilde{T}}=b(\tilde{T})[-F(x(\tilde{T}))+F(x(\tilde{T}-\sigma(\tilde{T})))] .
$$

It follows that

$$
x(\tilde{T}-\sigma(\tilde{T}))=x(\tilde{T}), z\left(s_{1}\right)=x(\tilde{T})-p x(\tilde{T}-\sigma(\tilde{T}))=(1-p) x(\tilde{T}-\sigma(\tilde{T})),
$$

which contradicts the facts that

$$
\begin{aligned}
& t-\sigma^{+}<\tilde{T}-\sigma(\tilde{T})<\tilde{T}-\sigma^{-} / 2<t \\
& (1-p) x(\tilde{T}-\sigma(\tilde{T}))=z\left(s_{1}\right)>z(t) \geq \max _{t-\sigma^{+} \leq s \leq t}(1-p) x(s) .
\end{aligned}
$$


Hence, (3.3) holds, and

$$
D^{+} z(t)=\limsup _{h \rightarrow 0^{+}} \frac{z(t+h)-z(t)}{h}=\limsup _{h \rightarrow 0^{+}} \frac{z(t)-z(t)}{h}=0, h \in\left(0, s_{1}-t\right] .
$$

Analogously, we can get

$$
D^{-} v(t) \geq 0 \text { for all } t>t_{0} .
$$

From the above results, we know that $x\left(t ; t_{0}, \varphi\right)$ is bounded on $\left[-\sigma^{+},+\infty\right)$, and

$$
\begin{aligned}
& \alpha=\limsup _{t \rightarrow \infty} \max \{(1-p) x(t), x(t)-p x(t-\sigma(t))\}=\lim _{t \rightarrow \infty} z(t)<+\infty \\
& \beta=\liminf _{t \rightarrow \infty} \min \{(1-p) x(t), x(t)-p x(t-\sigma(t))\}=\lim _{t \rightarrow \infty} v(t)>-\infty
\end{aligned}
$$

Finally, we prove that $x\left(t ; t_{0}, \varphi\right)$ tends to a constant as $t \rightarrow+\infty$. Let

$$
\alpha^{-}=\liminf _{t \rightarrow \infty} \max \{(1-p) x(t), x(t)-p x(t-\sigma(t))\} .
$$

It suffices to show that $\alpha^{-}=\alpha$. By way of contradiction, suppose that $\alpha^{-}<$ $\alpha$. Then $\alpha^{-}$and $\alpha$ are not zero simultaneously. Without loss of generality, we assume that $\alpha \neq 0$ since the proof for the case of $\alpha^{-} \neq 0$ is quite similar. For $H \in\left(\alpha^{-}, \alpha\right)$, we can choose $T_{0}^{*}>t_{0}$ and $\left\{r_{m}\right\}_{m=1}^{\infty} \subset\left[T_{0}^{*}+\sigma^{+},+\infty\right)$ such that

$$
\begin{aligned}
& z(t)=\max _{t-\sigma^{+} \leq s \leq t}\{x(s)-p x(s-\sigma(s)),(1-p) x(s)\} \leq \alpha+\frac{|\alpha|}{2} \forall t \in\left[T_{0}^{*},+\infty\right), \\
& \max \left\{(1-p) x\left(r_{m}\right), x\left(r_{m}\right)-p x\left(r_{m}-\sigma\left(r_{m}\right)\right)\right\}=H, \quad \lim _{m \rightarrow \infty} r_{m}=+\infty, \\
& 0 \leq \varepsilon_{m}=z\left(r_{m}\right)-\alpha \leq \frac{|\alpha|}{2}, \varepsilon_{m} \rightarrow 0(\text { as } m \rightarrow+\infty) .
\end{aligned}
$$

Let $w(t)=x(t)-p x(t-\sigma(t))$ for all $t \geq t_{0}$. It is clear that, for $t \in\left[r_{m}, r_{m}+2 \sigma^{+}\right]$,

$$
0 \leq-F\left(\frac{w(t)}{1-p}\right)+F\left(\frac{z\left(r_{m}\right)}{1-p}\right)=-F\left(\frac{w(t)}{1-p}\right)+F\left(\frac{\alpha+\varepsilon_{m}}{1-p}\right)
$$

and

$$
(1-p) x(t-\sigma(t)) \leq \max _{r_{m}-\sigma^{+} \leq t \leq r_{m}+2 \sigma^{+}}\{(1-p) x(t), w(t)\} \leq z\left(r_{m}\right)=\alpha+\varepsilon_{m} .
$$

This implies

$$
x(t-\sigma(t)) \leq \frac{\alpha+\varepsilon_{m}}{1-p} \text { for all } t \in\left[r_{m}, r_{m}+2 \sigma^{+}\right] .
$$

Now, we prove

$$
w^{\prime}(t) \leq b(t)\left[-F\left(\frac{w(t)}{1-p}\right)+F\left(\frac{\alpha+\varepsilon_{m}}{1-p}\right)\right], \text { for all } t \in\left[r_{m}, r_{m}+2 \sigma^{+}\right] .
$$

In fact, if $x(t)<x(t-\sigma(t))$ and $t \in\left[r_{m}, r_{m}+2 \sigma^{+}\right]$, then

$$
(1-p) x(t)>x(t)-p x(t-\sigma(t))=w(t), x(t)>w(t) /(1-p) .
$$


This implies that

$$
w^{\prime}(t)=b(t)[-F(x(t))+F(x(t-\sigma(t)))] \leq b(t)\left[-F\left(\frac{w(t)}{1-p}\right)+F\left(\frac{\alpha+\varepsilon_{m}}{1-p}\right)\right] .
$$

If $x(t) \geq x(t-\sigma(t))$ and $t \in\left[r_{m}, r_{m}+2 \sigma^{+}\right]$, then (3.4) implies that

$$
w^{\prime}(t)=b(t)[-F(x(t))+F(x(t-\sigma(t)))] \leq 0 \leq b(t)\left[-F\left(\frac{w(t)}{1-p}\right)+F\left(\frac{\alpha+\varepsilon_{m}}{1-p}\right)\right] .
$$

Thus, (3.6) always holds, and

$$
w\left(r_{m}\right) \leq H<\alpha
$$

Denote $q(t)=q\left(t ; r_{m}, \varepsilon_{m}\right)$ the solutions of the initial-value problem

$$
q^{\prime}(t)=b^{+}\left[-F\left(\frac{q(t)}{1-p}\right)+F\left(\frac{\alpha+\varepsilon_{m}}{1-p}\right)\right], \quad q\left(r_{m}\right)=H .
$$

Note that $H<\alpha$ and

$$
0 \leq b(t)\left[-F\left(\frac{w(t)}{1-p}\right)+F\left(\frac{\alpha+\varepsilon_{m}}{1-p}\right)\right] \leq b^{+}\left[-F\left(\frac{w(t)}{1-p}\right)+F\left(\frac{\alpha+\varepsilon_{m}}{1-p}\right)\right]
$$

for all $t \in\left[r_{m}, r_{m}+2 \sigma^{+}\right]$. By taking $G(x)=b^{+} F\left(\frac{x}{1-p}\right)$, Proposition 1 implies that

$$
\alpha+\varepsilon_{m}-q\left(t ; r_{m}, \varepsilon_{m}\right) \geq \mu>0, t \in\left[r_{m}, r_{m}+2 \sigma^{+}\right],
$$

where the positive constant $\mu$ is independent of $r_{m}$ and $\varepsilon_{m}$. Furthermore, from (3.6) and (3.7), we have

$$
w(t) \leq q(t)<\alpha+\varepsilon_{m}-\mu, t \in\left[r_{m}, r_{m}+2 \sigma^{+}\right]
$$

and there exists a positive integer $M$ such that

$$
\max _{s \in\left[r_{m}, r_{m}+2 \sigma^{+}\right]} w(s)<\alpha+\varepsilon_{m}-\mu<\alpha-\mu / 2, \text { for all } m>M .
$$

On the other hand, $D^{+} z(t) \leq 0$ produces that

$$
\max _{s \in\left[r_{m}, r_{m}+2 \sigma^{+}\right]}\{(1-p) x(s), w(s)\} \geq \alpha
$$

and thus there exists $r_{m}^{*} \in\left[r_{m}, r_{m}+2 \sigma^{+}\right]$such that $(1-p) x\left(r_{m}^{*}\right) \geq \alpha$. Hereafter, we have

$$
(1-p) x\left(r_{m}^{*}\right) \geq \alpha, \text { and } x\left(r_{m}^{*}\right) \geq \alpha /(1-p),
$$

which, together with (3.5), leads to

$$
\begin{aligned}
w\left(r_{m}^{*}\right)= & x\left(r_{m}^{*}\right)-p x\left(r_{m}^{*}-\sigma\left(r_{m}^{*}\right)\right) \\
& \geq \frac{\alpha}{1-p}-p \frac{\alpha+\varepsilon_{m}}{1-p}=\alpha-p \frac{\varepsilon_{m}}{1-p} \rightarrow \alpha(\text { as } m \rightarrow+\infty) .
\end{aligned}
$$


This is contrary to (3.8). Hence,

$$
\lim _{t \rightarrow \infty} \max \{(1-p) x(t), x(t)-p x(t-\sigma(t))\}=\alpha=\alpha^{-} .
$$

Similarly, we get

$$
\lim _{t \rightarrow \infty} \min \{(1-p) x(t), x(t)-p x(t-\sigma(t))\}=\beta .
$$

Consequently,

$$
\begin{aligned}
& \lim _{t \rightarrow \infty}\{(1-p) x(t)+x(t)-p x(t-\sigma(t))\}=\alpha+\beta, \\
& \lim _{t \rightarrow \infty}\left[x(t)-\frac{p x(t-\sigma(t))}{2-p}\right]=\frac{\alpha+\beta}{2-p} .
\end{aligned}
$$

It follows from Lemma 3 and the fact $\left|\frac{p}{2-p}\right|<1$ that $\lim _{t \rightarrow \infty} x(t)$ exists. This completes the proof.

\section{Conclusions}

Since the asymptotic behavior of NFDE with time-varying delays has not been touched in $[1,3,6,8,9,10,11,12,13,14,15]$, one can find that the analysis method of the dynamical systems in the above references cannot be applied to prove Theorem 1. Noting that $F(x)=x^{\frac{1}{3}}$ satisfies (1.5), it follows from Theorem 1 that the Haddock conjecture is true when for the delays are time-varying functions. Moreover, all results in [7] are the special cases where $b(t) \equiv 1$ and $\tau(t) \equiv r$ are constants. This implies that all proof of the main result in [1] can be improved by the analytical method in this paper. Thus, our results are essentially new and complement previously known ones to some extent.

\section{Acknowledgements}

The author is grateful to the anonymous reviewers for his/her thoughtful comments and constructive suggestions on improving our original manuscript. Also, I would like to express the sincere appreciation to Prof. Bingwen Liu (Jiaxing University) for the helpful discussion when this work was being carried out.

\section{References}

[1] H. Gao and H. Long. Dynamic behavior for a system of neutral functional differential equations. Computers \& Mathematics with Applications, 58(10):20462050, 2009. https://doi.org/10.1016/j.camwa.2009.07.056.

[2] J.R. Haddock. Functional differential equations for which each constant function is a solution: A narrative. In Proc. Eleventh Int. Conf. on Nonlinear Oscillations, M. Farkas, V. Kertesz, and G. Stepan, eds., Janos Bolyai Math. Soc., Budapest, pp. 86-93, 1987. 
[3] T. Krisztin and J. Wu. Asymptotic periodicity, monotonicity, and oscillation of solutions of scalar neutral functional differential equations. Journal of Mathematical Analysis and Applications, 199(2):502-525, 1996. https://doi.org/10.1006/jmaa.1996.0158.

[4] B. Liu. Asymptotic behavior of solutions to a class of non-autonomous delay differential equations. Journal of Mathematical Analysis and Applications, 446(1):580-590, 2017. https://doi.org/10.1016/j.jmaa.2016.09.001.

[5] B. Liu. A generalization of the Bernfeld-Haddock conjecture. Applied Mathematics Letters, 65:7-13, 2017. https://doi.org/10.1016/j.aml.2016.09.018.

[6] D. Tongren. Asymptotic behavior of solutions of some retarded differential equations. Science China Mathematics, 25(4):363-370, 1982.

[7] J. Wu. On J. R. Haddock's conjecture. Applicable Analysis, 33(1-2):127-137, 1989. https://doi.org/10.1080/00036818908839866.

[8] J. Wu. Asymptotic periodicity of solutions to a class of neutral functional differential equations. Proceedings of the American Mathematical Society, 113(2):355363, 1991. https://doi.org/10.2307/2048519.

[9] W. Xiong and B. Liu. Asymptotic behavior of bounded solutions for a system of neutral functional differential equations. Journal of Mathematical Analysis and Applications, 313(2):754-760, 2006. https://doi.org/10.1016/j.jmaa.2005.08.095.

[10] M. Xu, Z. Yuan, W. Wang and H. Long. $\omega$ limit sets of solutions for a class of neutral functional differential equations. Nonlinear Analysis: Real World Applications, 11(4):2345-2349, 2010. https://doi.org/10.1016/j.nonrwa.2009.07.008.

[11] M. Yang. Dynamic behavior for a class of neutral functional differential equations. Applied Mathematics Letters, 22(8):1185-1188, 2009. https://doi.org/10.1016/j.aml.2009.01.029.

[12] T. Yi. A generalization of the Haddock conjecture and its proof. Nonlinear Analysis: Real World Applications, 9(3):1112-1118, 2008. https://doi.org/10.1016/j.nonrwa.2007.02.006.

[13] T. Yi and G. Gu. Convergence of a class of discrete-time semiflows with application to neutral delay differential equations. Nonlinear Analysis: Theory, Methods \& Applications, 68(5):1148-1154, 2008. https://doi.org/10.1016/j.na.2006.12.011.

[14] T. Yi and L. Huang. Convergence for pseudo monotone semiflows on product ordered topological spaces. Journal of Differential Equations, 214(2):429-456, 2005. https://doi.org/10.1016/j.jde.2005.02.005.

[15] T.S. Yi and L.H. Huang. A generalization of the Bernfeld-Haddock conjecture and its proof. Acta Mathematica Sinica, Chinese Series, 50(2):261-267, 2007. 